\title{
Incidental findings from cancer next generation sequencing
} \section{panels}

Nika Maani ${ }^{1,2}$, Karen Panabaker ${ }^{3}$, Jeanna M. McCuaig ${ }^{2,4}$, Kathleen Buckley ${ }^{5}$, Kara Semotiuk ${ }^{6}$, Kirsten M. Farncombe ${ }^{7}$, Peter Ainsworth ${ }^{8}$, Seema Panchal ${ }^{2,9}$, Bekim Sadikovic ${ }^{8,10}$, Susan Randall Armel ${ }^{2,4}$, Hanxin Lin $\mathbb{D}^{8,10,12 \bowtie}$ and Raymond H. Kim (D) ${ }^{4,11,12 \bowtie}$

Next-generation sequencing (NGS) technologies have facilitated multi-gene panel (MGP) testing to detect germline DNA variants in hereditary cancer patients. This sensitive technique can uncover unexpected, non-germline incidental findings indicative of mosaicism, clonal hematopoiesis $(\mathrm{CH})$, or hematologic malignancies. A retrospective chart review was conducted to identify cases of incidental findings from NGS-MGP testing. Inclusion criteria included: 1) multiple pathogenic variants in the same patient; 2) pathogenic variants at a low allele fraction; and/or 3) the presence of pathogenic variants not consistent with family history. Secondary tissue analysis, complete blood count $(\mathrm{CBC})$ and medical record review were conducted to further delineate the etiology of the pathogenic variants. Of 6060 NGS-MGP tests, 24 cases fulfilling our inclusion criteria were identified. Pathogenic variants were detected in TP53, ATM, CHEK2, BRCA1 and APC. 18/24 (75.0\%) patients were classified as CH, 3/24 (12.5\%) as mosaic, 2/24 $(8.3 \%)$ related to a hematologic malignancy, and $1 / 24(4.2 \%)$ as true germline. We describe a case-specific workflow to identify and interpret the nature of incidental findings on NGS-MGP. This workflow will provide oncology and genetic clinics a practical guide for the management and counselling of patients with unexpected NGS-MGP findings.

npj Genomic Medicine (2021)6:63; https://doi.org/10.1038/s41525-021-00224-6

\section{INTRODUCTION}

Blood-based genetic testing is a mainstay in the approach of hereditary cancer assessment ${ }^{1-7}$. Such genetic testing typically involves the analysis of DNA isolated from peripheral blood leukocytes (PBLs) $)^{3,8,9}$, with the assumption that leukocyte DNA is representative of the germline ${ }^{8-10}$. Most hereditary cancer syndromes are inherited in a monoallelic fashion with an expected $50 \%$ allele fraction $(\mathrm{AF})^{8}$. Compared to traditional Sanger sequencing, next generation sequencing (NGS) technologies and the development of multi-gene panel (MGP) testing have significantly improved assay sensitivity and enabled the ability to test multiple genes simultaneously ${ }^{4,8,9,11}$. In a minority of cases, atypical genetic test results can be observed following NGS-MGP testing, which are not consistent with a monoallelic germline variant. Examples include the detection of variants with low AFs $(<30 \%)^{3,8,9,12}$, multiple pathogenic variants in the same individual, and genetic testing results which are not congruent with the reported family history ${ }^{13,14}$. When derived from the analysis of PBLs, these unexpected genetic results may indicate a de novo germline event ${ }^{15}$, or a genetic variant of non-germline origin. These results could be considered incidental findings ${ }^{16}$, as they are outside of the original purpose for which the test or procedure was conducted $^{17}$. Non-germline findings include post-zygotic mosaicism, hematologic malignancy, or clonal hematopoesis $(\mathrm{CH})$ (a newly recognized phenomenon where genetic changes accumulate in myeloid cells) $)^{3,5,6,8}$.

$\mathrm{CH}$ is a recently described phenomenon in which somatic variants accumulate in PBL due to aging and exposure to therapies such as chemotherapy and radiotherapy ${ }^{8,18-20}$. These somatic variants can lead to precursors for neoplasms of undetermined significance ${ }^{18,21-23}$. Clinically, a growing body of evidence has uncovered a link between $\mathrm{CH}$ and a two to four-fold increased risk of atherosclerotic disease ${ }^{18,24-28}$, along with an increased risk for the development of overt hematologic neoplasia and increased overall mortality in $\mathrm{CH}$ patients ${ }^{29}$. Mosaicism is typically defined as the presence of genetically distinct populations of cells within an individual, following acquired change(s) occurring shortly after zygote formation ${ }^{30,31}$. If these changes are present in the gametes and involve germline cells, they may be transmissible to offspring 9,10,30-32. Postnatally, further somatic tissue-specific genetic changes can be acquired and can lead to the development of cancer ${ }^{8-10}$. When somatic variants are detected exclusively in PBLs, they can be indicative of a hematological malignancy or $\mathrm{CH}^{8,9,33}$ and may lead to clonal expansion in the blood $^{21,34}$. PBL-isolated variants, however, are not transmissible to offspring and their identification is important as patients carrying such variants are managed differently from those harbouring germline variants.

Given the increased uptake of NGS-MGP testing, there exists a growing need for the development of a workflow to both identify and manage the clinical follow-up of patients who present with potentially non-germline incidental findings. To further explore the management of patients presenting with potential incidental findings following germline NGS-MGP testing, we performed a retrospective chart review of individuals who underwent hereditary cancer NGS-MGP testing in Ontario with findings suspicious of a non-germline incidental finding and propose a clinical workup.

\footnotetext{
${ }^{1}$ Program for Genetics and Genome Biology, Hospital for Sick Children, Toronto, ON, Canada. ${ }^{2}$ Department of Molecular Genetics, University of Toronto, Toronto, ON, Canada. ${ }^{3}$ Medical Genetics Program of Southwestern Ontario, London Health Sciences Centre, London, ON, Canada. ${ }^{4}$ Familial Cancer Clinic, Princess Margaret Hospital Cancer Centre, Toronto, ON, Canada. ${ }^{5}$ Grand River Hospital, Kitchener, ON, Canada. ${ }^{6}$ Zane Cohen Centre for Digestive Diseases, Familial Gastrointestinal Cancer Registry, Mount Sinai Hospital, Toronto, ON, Canada. ${ }^{7}$ Toronto General Hospital Research Institute, University Health Network, Toronto, ON, Canada. ${ }^{8}$ Molecular Genetics Laboratory, Division of Molecular Diagnostics, London Health Sciences Centre, London, ON, Canada. ${ }^{9}$ Familial Breast Cancer Clinic, Mount Sinai Hospital, Toronto, ON, Canada. ${ }^{10}$ Department of Pathology and Laboratory Medicine, Western University, London, ON, Canada. ${ }^{11}$ Department of Medicine, University of Toronto, Toronto, ON, Canada. ${ }^{12}$ These authors contributed equally: Hanxin Lin, Raymond H. Kim. ${ }^{凶}$ email: hanxinlin@lhsc.on.ca; raymond.kim@uhn.ca
} 


\section{RESULTS}

\section{Patient population}

Between January 1, 2016 and December 31, 2019, 6060 patients with a personal or strong family history of cancer who were referred to a hereditary cancer clinic in Ontario on suspicion of a hereditary cancer syndrome consented to have NGS-MGP testing. All 6060 patients underwent a germline comprehensive hereditary cancer NGS panel at the Molecular Genetics Laboratory at London Health Sciences Centre in Ontario, Canada. Of these 6060 patients, 24 (0.4\%) fulfilled the inclusion criteria suggestive of an incidental finding (Table 1). For patients with unquantified low allele fractions from an external laboratory (Patients 2, 33, 40), NGS-MGP was repeated at London Health Sciences Centre. To better understand the origin of these variants, NGS-MGP was conducted on secondary tissues and blood collected at different time points. This secondary testing was done in conjunction with a review of the patient's medical history (including complete blood counts $[\mathrm{CBCs}]$ ) to monitor for the presence or potential development of a hematologic disorder.

\section{Genetic testing results}

Twenty-four patients fulfilled the inclusion criteria and underwent further investigation of secondary tissues and an assessment for $\mathrm{CH}$ and leukemia (Table 2). Four out of twenty-four (16.6\%) patients had multiple pathogenic variants (Category 1), 18/24 (75\%) patients had low AFs (Category 2), and 17/24 (70\%) patients whose family histories were not suggestive of Li-Fraumeni syndrome (non-Chompret) had TP53 variants (Category 3). Of note, many patients fit into more than one category. Identified cases involved 5 different hereditary cancer genes; the most common being TP53. Of the 24 patients in our cohort, 17 (70\%) had findings in TP53, 2 (8.3\%) in CHEK2, 3 (12.5\%) in ATM, 2 (8.3\%) in APC and 2 (8.3\%) in BRCA1. Two out of twenty-four $(8.3 \%)$ patients had pathogenic variants in more than one gene. To understand the origin of these variants, peripheral blood was collected at different time points when possible, and multiple AFs were obtained. Heterozygous variants were expected to have an AF of approximately 50\%; however, in the case of non-germline variants, the AF may significantly fluctuate.

\section{Secondary tissue analysis}

Fibroblast culturing has been reported to boost allelic fractions of variants that confer a growth advantage, suggesting that fibroblasts harbouring pathogenic variants in tumour suppressor genes may be present in cultured samples ${ }^{35}$. To further delineate this in our cohort, patients underwent a skin biopsy (direct and/or cultured). Eleven out of twenty-four (45.8\%) patients underwent NGS-MGP analysis of a direct skin punch biopsy. Of these, 7 $(63.6 \%)$ had a paired cultured skin biopsy. Four out of eleven (36.3\%) patients $(11,12,13$, and 18) harboured low levels of PBL variants in direct skin biopsy samples and upon fibroblast culturing, these variants were no longer observed (Table 2). This suggests PBL contamination of the direct skin punch biopsies and is suggestive of $\mathrm{CH}$. This was also observed in Patient 10 , in which an additional second and third pathogenic variant in TP53 were identified in direct skin punch biopsy. These variants were different than those initially identified in PBL-derived DNA and in cultured fibroblasts (Table 2), suggesting the presence of sequencing artifacts or somatic events occurring in the skin. Sample mix-up was ruled out by the assessment of three unique SNPs on skin and blood samples and results were confirmed using an additional NGS chemistry and sequencer. Taken together, the two additional variants found in the direct skin biopsy were likely somatic events isolated to the skin which have been observed in healthy individuals ${ }^{36-38}$. Conversely, Patient 6 harboured low levels of a TP53 variant in direct skin punch biopsy, suggestive of PBL contamination in the biopsy sample. A cultured fibroblast sample for this patient was not available and it was ultimately concluded that this patient likely had $\mathrm{CH}$ (Table 2).

Three out of twenty-four (12.5\%) patients underwent tumour tissue analysis. Patient 40 was mosaic for APC; patient 41 had a TP53 variant present in the tumour at a low level, but did not have the second APC variant (Table 2). This case was considered likely due to $\mathrm{CH}$ and the tumour results due to the presence of admixed leukocytes of $\mathrm{CH}$ origin in tumour tissues. This phenomenon has also been reported in other tumour sequencing studies ${ }^{5,22}$. Lastly, skin and tumour samples in Patient 3 were negative for the TP53 variant detected in $\mathrm{PBL}$, suggesting $\mathrm{CH}$.

\section{Diagnostic algorithm and conclusions}

Diagnostic conclusions for patients in our cohort were made using a specific algorithm (Fig. 1). Patients were considered to have a confirmed full germline hereditary cancer syndrome if the variant was found at $>30 \%$ AF in PBL and cultured fibroblasts [1/24 $(4.2 \%)]$. Patients were classified as mosaic if the variant was detected at $\mathrm{a}<30 \% \mathrm{AF}$ in PBL-derived DNA and was also detected in a second tissue [3/24 (12.5\%)]. Mosaic patients were clinically managed using relevant hereditary cancer syndrome screening guidelines.

Nine out of twenty-four (37.5\%) patients had a variant with an AF ( $<30 \%$ ) in PBL, which was either absent, or present in low levels in secondary tissues (results from cultured fibroblasts were used if there was a discrepancy with direct skin biopsy results). These patients were further evaluated for $\mathrm{CH}$ or a hematologic malignancy through a thorough review of $C B C$ and medical charts (Fig. 1). If the $C B C$ was normal and there was no evidence of hematologic malignancy upon medical chart review, patients were classified as $\mathrm{CH}$ [8/24 (33.3\%)] (Fig. 1, Table 2). Molecular findings such as low AFs, normal CBC and exposure to chemotherapy were considered to support a diagnosis of $\mathrm{CH}$. Fluctuating AF was also supportive of $\mathrm{CH}$, however, this finding alone may be insufficient evidence of $\mathrm{CH}$ as the variance between timepoints was not assessed. Higher AFs and positive family history were considered suggestive of an inherited syndrome. Under this criteria, an additional 10 patients were classified as "likely $\mathrm{CH}^{\prime}$ based on clinical history despite not having secondary tissue analysis. Combining these 10 patients with the $8 \mathrm{CH}$ cases confirmed through secondary tissue analysis, 18/24 (75.0\%) patients were concluded to have $\mathrm{CH}$, representing a majority of our cohort. Of interest, $14 / 18 \mathrm{CH}$ patients $(77.7 \%)$ had a history of chemotherapy (Tables 1 and 2). In keeping with the ever-evolving clinical impact of $\mathrm{CH}$, patients with findings suggestive of $\mathrm{CH}$ were enrolled into a research program assessing cardiovascular risk reduction ${ }^{18,24-28}$.

Lastly, 2 out of $24(8.3 \%)$ patients were diagnosed with a hematologic malignancy. Patient 11 had an elevated white blood cell count and was diagnosed with chronic lymphoblastic leukemia whilst simultaneously undergoing germline testing. Patient 12 did not disclose their multiple myeloma diagnosis during the initial genetics consultation. Upon further review of their medical chart, it was noted they had been lost to follow-up regarding this diagnosis and was subsequently re-referred to their hematologist for ongoing care. These cases highlight the importance of a thorough medical history review for all patients undergoing a genetics assessment.

\section{DISCUSSION}

This retrospective chart review investigated incidental findings identified through NGS-MGP testing for hereditary cancer syndrome identification. These findings were considered incidental as they were unexpected from the original reason for NGS-MGP ${ }^{17}$. Genetic findings meriting further investigation for an incidental finding include: (1) multiple pathogenic variants in the same individual, (2) low AFs, and (3) pathogenic variants not consistent with personal and/or family cancer history (e.g. non-Chompret ${ }^{8,39}$ 


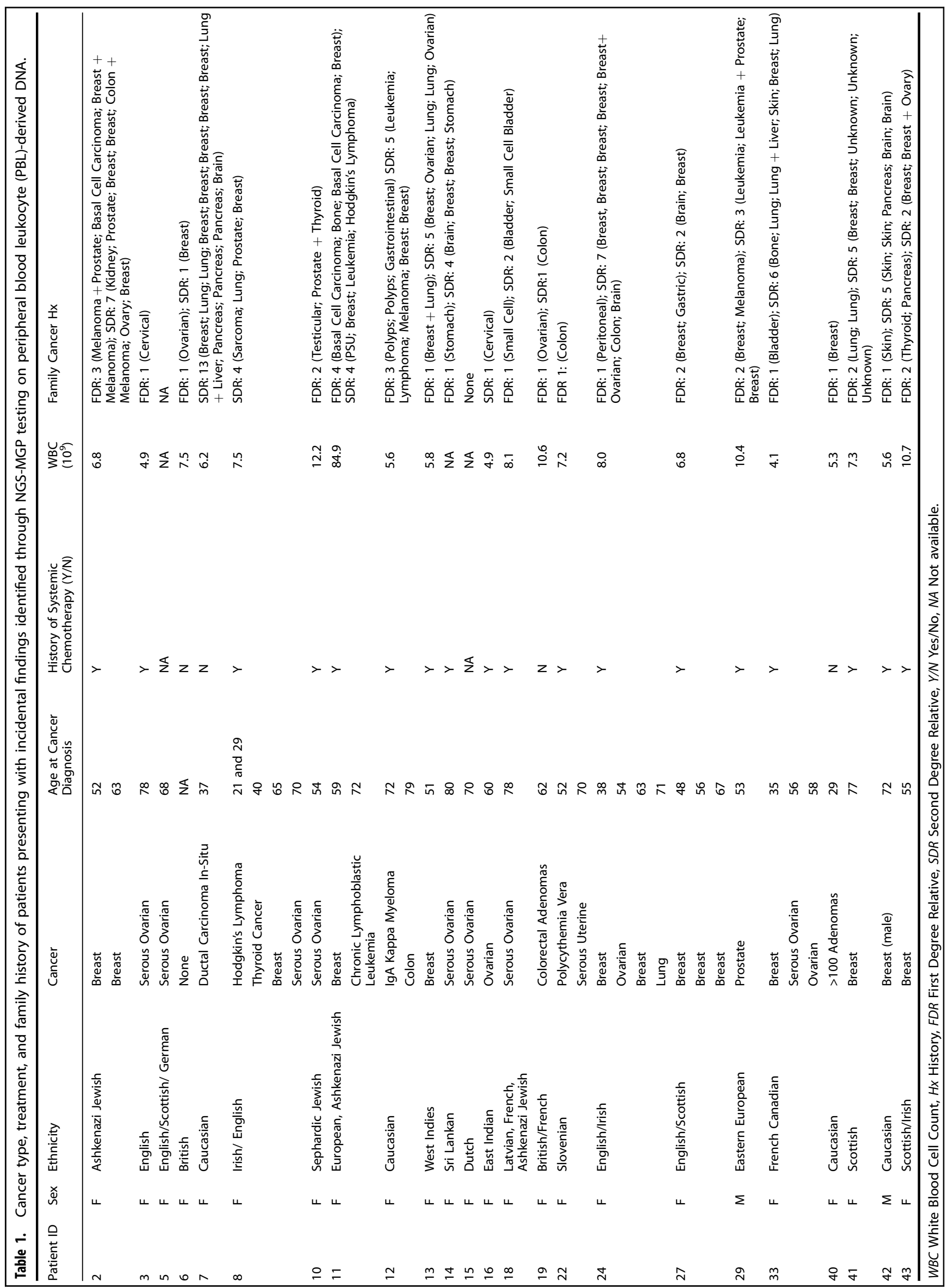




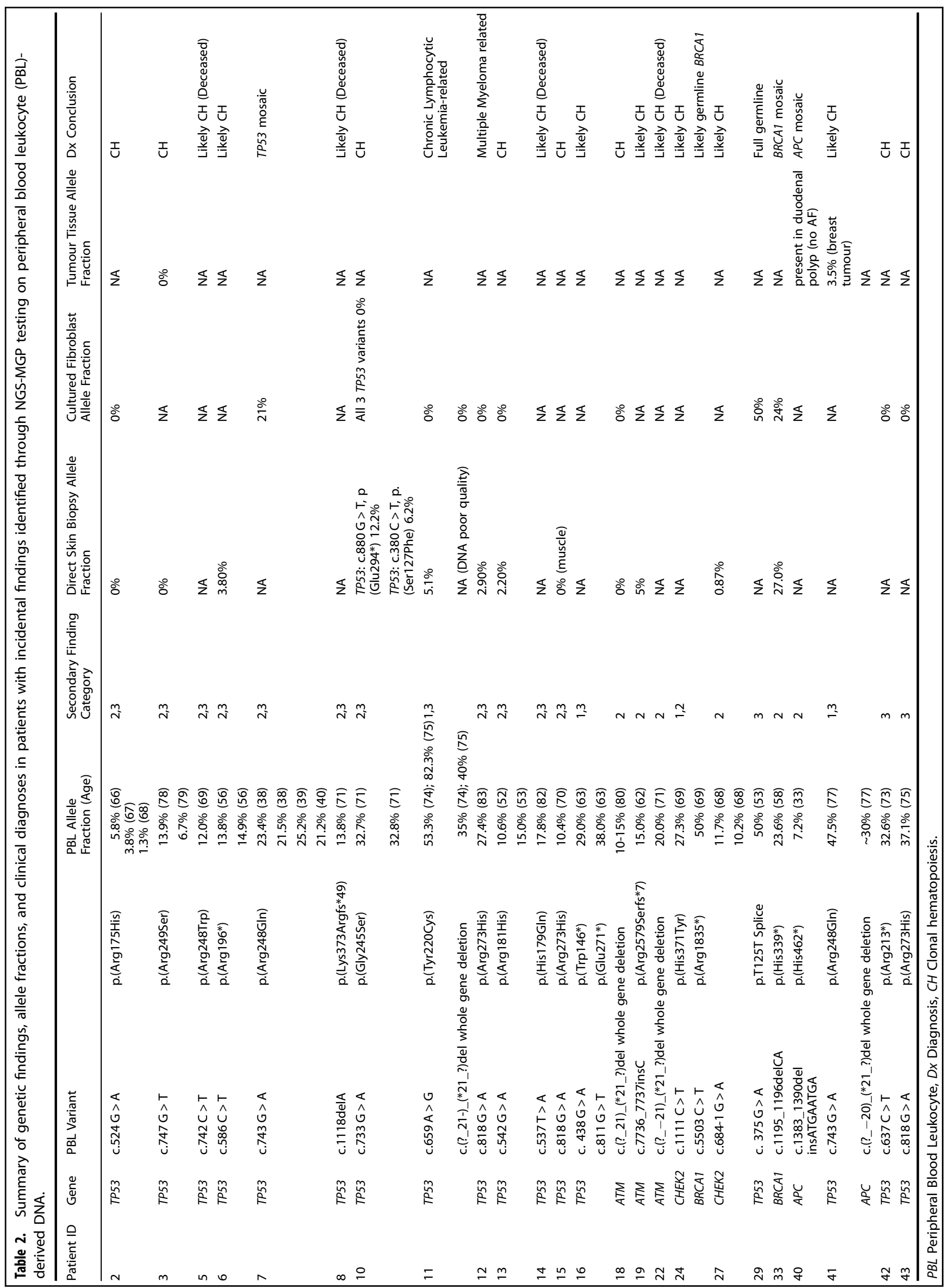




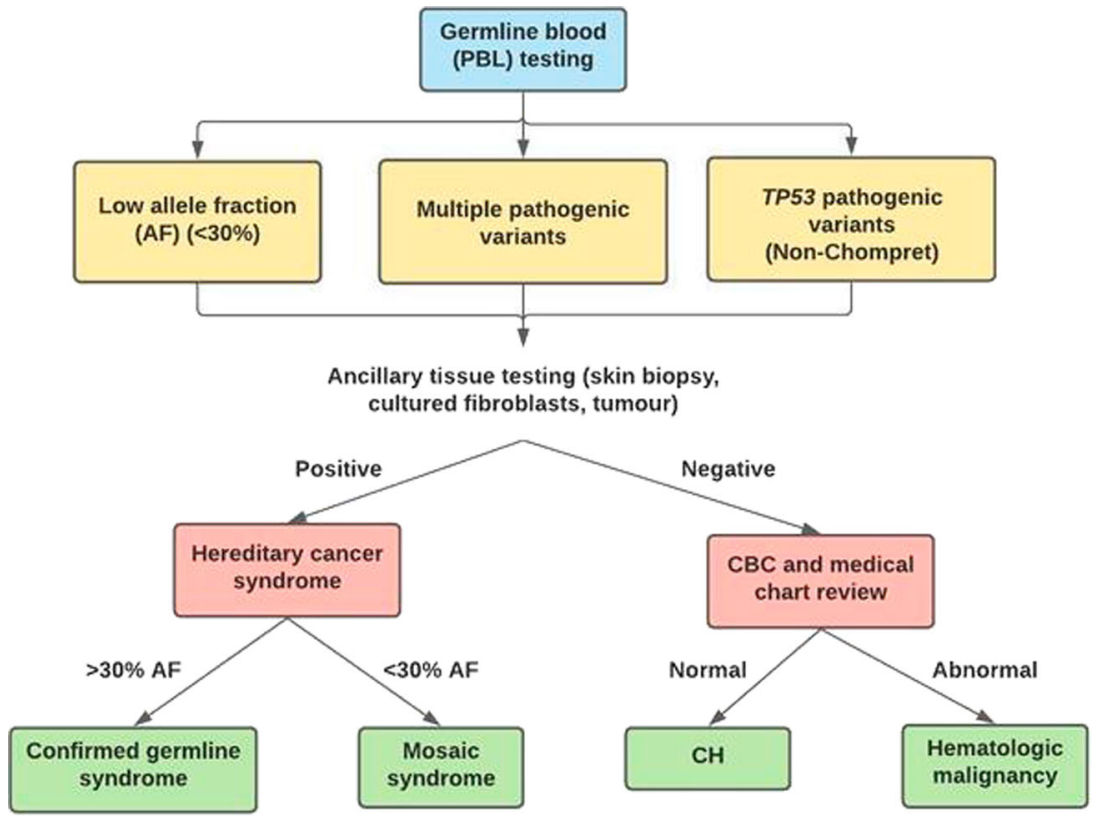

Fig. 1 Genetic findings. Recommended diagnostic algorithm for patients presenting with potential incidental findings identified through NGS-MGP testing on peripheral blood leukocyte (PBL)-derived DNA. Genetic findings in individuals undergoing germline NGS-MGP testing on PBLs are classified in one of three categories and subsequently undergo further testing to identify downstream surveillance and diagnosis. This includes secondary tissue analysis (culture skin biopsy preferred) and medical chart review. CBC complete blood count.

families harbouring TP53 variants). We propose a clinical algorithm to determine the type of incidental finding and management of these cases (Fig. 1).

Aspects of our clinical workflow include secondary tissue analysis (cultured fibroblasts preferred) and a thorough review of $\mathrm{CBC}$ and medical records, builds on previously published algorithms ${ }^{8}$. The clinical implications of incidental findings can be significant and delineating their origin may directly impact patient management. Patients harbouring germline and mosaic pathogenic variants need to undergo annual radiological surveillance and/or surgical intervention. This is particularly relevant for TP53 variants, which is the only gene on the NGS-MGP that is clearly associated with $\mathrm{CH}$ and overlaps with the highly penetrant hereditary Li-Fraumeni syndrome.

Based on their constellation of clinical findings, the majority of patients in our cohort were diagnosed as $\mathrm{CH}$ (33.3\%) or likely $\mathrm{CH}$ (41.6\%). Although $\mathrm{CH}$ is not considered a hematologic disorder, it represents a risk factor and a potential precursor for the evolution of various hematologic malignancies (myelodysplastic syndrome (MDS) and acute myeloid leukemia $(\mathrm{AML}))^{40}$, as well as cardiovascular complications ${ }^{18,24-28}$. Indeed, this emphasizes the importance of identifying and coordinating appropriate follow-up for $\mathrm{CH}$ patients, particularly those who have undergone chemotherapy. Of note, one $\mathrm{CH}$ patient in our cohort (Patient 6) was young (56y) and had no history of chemotherapy or known cancer diagnosis at the time of testing (Table 1, Table 2). While rare, $\mathrm{CH}$ has been observed in healthy individuals $<50$ years of age ${ }^{41}$, demonstrating the importance of additional investigations for all patients with potential incidental findings, irrespective of age or cancer history.

In emphasizing certain cases, we seek to demonstrate complexities in the work-up of these patients, including some challenges in secondary tissue testing such as the limitations of direct skin punch biopsies and tumour tissue testing. These included, but were not limited to, the presence of somatic variants in the skin (Patient 10) and PBL contamination (Patients 12 and 13). Limitations of tumour testing included tissue infiltration of lymphocytes (Patient 41) and unreliable AFs (Patient 40). Indeed, streamlining secondary tissue testing is often complicated by logistical barriers in diagnostic laboratories. While many laboratories lack the appropriate resources to perform secondary tissue testing, the cases demonstrated here support the analysis of secondary tissues in certain clinical scenarios. This could include buccal swabs as an alternative to skin biopsy to distinguish $\mathrm{CH}$ from germline variants ${ }^{42}$.

The interpretation of incidental findings identified through NGSMGP should be undertaken with high clinical suspicion, thorough genetic counselling and in communication with the molecular genetics laboratory and the patient. An example of this process is emphasized through a review of Patient 41 . This case involved a family where genetic analysis of the proband identified an apparently heterozygous TP53 gene mutation at an AF of $47.5 \%$, a finding which was initially reported as a germline variant (Table 2). Upon genetic counsellor review, the family history did not fulfill Chompret's Criteria $^{8,39}$ and it became apparent that this result was not consistent with Li-Fraumeni Syndrome, likely representing an incidental finding. Through subsequent review of NGS-MGP data, an additional deletion in APC was identified at $30 \%$ allele fraction, strongly suggesting that the apparently heterozygous TP53 variant was likely not germline. This was confirmed by the absence of the TP53 variant in a secondary tissue (Table 2). Given that Patient 41 had no history of polyposis and was not initially counselled for the analysis of $A P C$, this scenario emphasizes the importance of reviewing the patient's cancer pedigree in context with genetic testing results. Genetic testing data that are secondary to the initial indication for testing may be of significant value in the interpretation of the final result for the patient. With the widespread use of NGS-MGP testing, analogous scenarios will become increasingly prevalent. Genetic counselling should involve a discussion of potential incidental results and thus raise awareness of the clinical implications.

Incidental findings represent a growing and unintended consequence of NGS-MGP testing in cancer patients. There exists a pressing need to properly advise patients of the different risks associated with incidental findings and the impact they may have on future care for themselves and their relatives. This includes patients with an atypical presentation of a germline hereditary cancer syndrome, post-zygotic mosaicism, $\mathrm{CH}$, or hematologic malignancy. True germline and mosaic cases have implications for cancer risk in 
patients and their family members. In contrast, hematologic malignancies and $\mathrm{CH}$ require significantly different medical management strategies and are unlikely to affect family members. Moreover, with limited understanding of the etiology and significance of incidental findings, genetic testing of these variants should be offered to all offspring and other family members to determine transmission of the variant and assess segregation ${ }^{13}$.

Given that the use of NGS-MGP testing for cancer susceptibility genes has increased, the identification of incidental findings will become more pervasive amongst genetics clinics. To address this, we propose the adoption of a clinical workflow that will streamline the management of these patients. It is anticipated that future larger studies will demonstrate the impact of this workflow in wider populations.

\section{METHODS}

\section{Patient population}

A retrospective chart review of patients who underwent NGS-MGP testing was conducted to identify genetic testing results suggestive of a non-germline incidental finding (mosaic, $\mathrm{CH}$, hematologic malignancy). Patients with a personal and/or strong family history of hereditary cancer who underwent germline NGS-MGP testing at the clinicial diagnostic laboratory at London Health Sciences Centre between January 1, 2016 and December 31, 2019 were reviewed. The inclusion criteria were: Category 1) multiple pathogenic or likely pathogenic variants in the same individual; Category 2) presence of likely pathogenic or pathogenic variants at low AFs (10-30\%); and/or Category 3) patients harbouring pathogenic TP53 variants not fulfilling any of the criteria for LFS (i.e. Chompret criteria ${ }^{8,39}$ ). Of note, three patient samples (Patients 2, 33, 40) were initially tested at an external laboratory and NGS-MGP was repeated at London Health Sciences Centre. Participants not meeting the inclusion criteria were excluded. Ethical approval was obtained from all sites by the Research Ethics Committees (University Health Network REB: 18-5484; London Health Sciences Centre REB: R-20-354; Sinai Health System REB: 030231-U). A waiver of consent was provided by all three Research Ethics Committees.

\section{Data collection and analysis}

For patients fulfilling an inclusion criteria, secondary tissues and/or blood collected at different time points were obtained when available for NGSMGP testing. Secondary tissues included skin biopsy (with or without fibroblast culture), other normal tissues (e.g. muscle) and tumour tissue. Secondary tissue analysis was not conducted on patients who did not fulfill the inclusion criteria as their NGS-MGP results were not considered unexpected. A review of medical records was completed, including hematology, pathology and screening reports. Collected information included type of genetic testing, gene variant, date of referral for genetic testing, date of consultation, as well as cancer diagnoses, including modality of detection, pathology and treatment. Descriptive statistics were used to describe clinical findings in the patient population.

\section{Genetic testing}

All genetic testing was done in a clinical molecular genetics laboratory (London Health Sciences Centre) and allele fractions were reported on all cases.

\section{DNA extraction}

Genomic DNA from blood samples were isolated by standard protocols (MagNA Pure system, Roche Diagnostics, Laval, QC, Canada). DNA was quantified through absorbance with a DTX 880 Multimode Detector (Beckman Coulter, Brea, CA).

\section{Next-generation sequencing analysis}

Genes included on the NGS-MPG panel include: APC (incl. 5'UTR), ATM, BARD1, BMPR1A, BRCA1, BRCA2, BRIP1, CDH1, CDK4, CDKN2A, CHEK2, CTNNA1, EPCAM, FANCC, FANCM, FLCN, GREM1, HOXB13, MEN1, MLH1 (incl. 5'UTR), MSH2, MSH3, MSH6, MUTYH, NBN, NTHL1, PALB2, PMS2, POLE, POLD1, PTEN (incl. 5'UTR), RAD51C, RAD51D, SDHB, SMAD4, STK11, TP53. All coding exons and 20 base pairs of flanking non-coding sequences were enriched using a custom targeted hybrid protocol. Libraries were prepared with $100 \mathrm{ng}$ of genomic
DNA fragmented to 180 to $220 \mathrm{bp}$ using a Covaris E220 Series Focusedultrasonicator (Covaris, Inc., Woburn, MA). Each sample library was ligated with a specific barcode index according to the manufacturer's protocol (Roche NimbleGen, Inc., Madison, WI) and assessed for quantification and size distribution using the Qubit fluorometer (Life Technologies) and 2200 TapeStation (Agilent Technologies, Santa Clara, CA) respectively. DNA libraries were pooled as 24-plex and captured using the SeqCap EZ Choice Library system (Roche NimbleGen, Inc.). Captured libraries underwent appropriate quality control analysis and were diluted to a concentration of $4 \mathrm{nmol} / \mathrm{L}$ to process for sequencing according to the manufacturer's instructions (Illumina, San Diego, CA). Library concentration for sequencing was $10 \mathrm{pmol} / \mathrm{L}$ with a $1 \%$ PhiX spike-in. Libraries were sequenced using the MiSeq version 2 reagent kit, generating $2 \times 150 \mathrm{bp}$ paired-end reads using the MiSeq fastq generation mode (lllumina). The average coverage of the panel was between 500-1000x. For the direct skin biopsy for patient 10, to further assess the TP53 variants found in the skin, a second NGS chemistry (Thermo-Fisher solid tumour Hotspot NGS panel) and second NGS sequencer (Ion Torrent S5) was used. Sequence alignment, variant calling and viewing were performed by the NextGENe and Geneticist Assistant software (Softgenetics). Variant interpretation and classification followed ACMG guidelines. Allele fractions of $30 \%$ were considered the empirical low threshold for heterozygous calling. This was established based on validation conducted on single nucleotide variants from NGS-MGP panels. While less reliable for copy number variants, a similar allele fraction threshold was used for exon-level deletions and MLPA was used to confirm (below). NGS allele fractions of $30 \%-70 \%$ were considered heterozygous.

\section{Multiplex ligation dependent probe amplification (MLPA) Analysis}

For patients with exon-level deletions on NGS $(11,18,22,41)$ MPLA was used to confirm these findings. Genomic DNA (100 ng) was amplified according to the manufacturer's recommendations (SALSA MLPA kit, MRC Holland, Amsterdam, the Netherlands). PCR products were separated by capillary electrophoresis (ABI 3730, Life Technologies, Thermo Fisher Scientific, Waltham, MA). Copy number alterations were analyzed with Coffalyser. Net software version 131211.1524 (MRC Holland).

\section{Sanger sequencing}

Sanger sequencing was used for all cases to confirm the SNVs or small indels identified by NGS panel with $>5 \%$ of allele fractions. Coding regions and flanking intronic regions $(-20 \mathrm{bp}$ to $+10 \mathrm{bp}$ ) were PCR-amplified and sequenced (BigDye Terminator v1.1 cycle sequencing kit, Life Technologies). Sequencing products were separated by capillary electrophoresis on an ABI3730 (Life Technologies) and analyzed with Mutation Surveyor v4.0.7 software (SoftGenetics).

\section{Reporting summary}

Further information on research design is available in the Nature Research Reporting Summary linked to this article.

\section{DATA AVAILABILITY}

The data that support the findings of this study are available on request from the corresponding author. Raw next-generation sequencing data has been deposited in Sequence Read Archive (SRA): SUB9747032, BioProject \#PRJNA734018.

Received: 31 December 2020; Accepted: 9 June 2021; Published online: 19 July 2021

\section{REFERENCES}

1. Meldrum, C., Doyle, M. A. \& Tothill, R. W. Next-generation sequencing for cancer diagnostics: a practical perspective. Clin. Biochem. Rev. 32, 177-195 (2011).

2. Guidugli, L. et al. Clinical utility of gene panel-based testing for hereditary myelodysplastic syndrome/acute leukemia predisposition syndromes. Leukemia 31, 1226-1229 (2017).

3. Coffee, B. et al. Detection of somatic variants in peripheral blood lymphocytes using a next generation sequencing multigene pan cancer panel. Cancer Genet. 211, 5-8 (2017). 
4. Nagahashi, M. et al. Next generation sequencing-based gene panel tests for the management of solid tumors. Cancer Sci. 110, 6-15 (2019).

5. Coombs, C. C. et al. Identification of clonal hematopoiesis mutations in solid tumor patients undergoing unpaired next-generation sequencing assays. Clin. Cancer Res. 24, 5918-5924 (2018).

6. DiNardo, C. D. et al. Improving the detection of patients with inherited predispositions to hematologic malignancies using next-generation sequencing-based leukemia prognostication panels. Cancer 124, 2704-2713 (2018).

7. Robson, M. E. et al. American society of clinical oncology policy statement update: genetic and genomic testing for cancer susceptibility. J. Clin. Oncol. 33, 3660-3667 (2015).

8. Batalini, F. et al. Li-Fraumeni syndrome: not a straightforward diagnosis anymore -the interpretation of pathogenic variants of low allele frequency and the differences between germline PVs, mosaicism, and clonal hematopoiesis. Breast Cancer Res. 21, 107 (2019).

9. Cohen, A. S. A., Wilson, S. L., Trinh, J. \& Ye, X. C. Detecting somatic mosaicism: considerations and clinical implications. Clin. Genet. 87, 554-562 (2015)

10. Freed, D., Stevens, E. L. \& Pevsner, J. Somatic mosaicism in the human genome. Genes 5, 1064-1094 (2014).

11. Beck, T. F., Mullikin, J. C., NISC Comparative Sequencing Program \& Biesecker, L. G. Systematic evaluation of sanger validation of next-generation sequencing variants. Clin. Chem. 62, 647-654 (2016).

12. Slavin, T. P. et al. Prevalence and characteristics of likely-somatic variants in cancer susceptibility genes among individuals who had hereditary pan-cancer panel testing. Cancer Genet. 235-236, 31-38 (2019).

13. Weitzel, J. N. et al. Somatic TP53 variants frequently confound germ-line testing results. Genet. Med. 20, 809-816 (2018).

14. Mester, J. L. et al. Apparently heterozygous TP53 pathogenic variants may be blood limited in patients undergoing hereditary cancer panel. Test. J. Mol. Diagn. JMD 22, 396-404 (2020).

15. Renaux-Petel, M. et al. Contribution of de novo and mosaic TP53 mutations to LiFraumeni syndrome. J. Med. Genet. 55, 173-180 (2018).

16. Tan, N. et al. Is 'incidental finding' the best term?: a study of patients' preferences. Genet. Med. 19, 176-181 (2017).

17. Cushman-Vokoun, A. et al. Laboratory and clinical implications of incidental and secondary germline findings during tumor testing. Arch. Pathol. Lab. Med. https:// doi.org/10.5858/arpa.2020-0025-CP.

18. Dorsheimer, L. et al. Association of mutations contributing to clonal hematopoiesis with prognosis in chronic ischemic heart failure. JAMA Cardiol. 4, 25 (2019).

19. Steensma, D. P. Clinical implications of clonal hematopoiesis. Mayo Clin. Proc. 93, 1122-1130 (2018).

20. Coombs, C. C. et al. Therapy-related clonal hematopoiesis in patients with nonhematologic cancers is common and associated with adverse clinical outcomes. Cell Stem Cell 21, 374-382.e4 (2017).

21. Boettcher, S. \& Ebert, B. L. Clonal hematopoiesis of indeterminate potential. J. Clin. Oncol. 5.

22. Ptashkin, R. N. et al. Prevalence of clonal hematopoiesis mutations in tumor-only clinical genomic profiling of solid tumors. JAMA Oncol. 4, 1589 (2018).

23. Xie, M. et al. Age-related mutations associated with clonal hematopoietic expansion and malignancies. Nat. Med. 20, 1472-1478 (2014).

24. Jaiswal, S. et al. Clonal hematopoiesis and risk of atherosclerotic cardiovascular disease. N. Engl. J. Med. 377, 111-121 (2017).

25. Jaiswal, S. et al. Age-related clonal hematopoiesis associated with adverse outcomes. N. Engl. J. Med. 371, 2488-2498 (2014).

26. Gillis, N. K. et al. Clonal haemopoiesis and therapy-related myeloid malignancies in elderly patients: a proof-of-concept, case-control study. Lancet Oncol. 18, 112-121 (2017).

27. Libby, P. \& Ebert, B. L. CHIP (Clonal Hematopoiesis of Indeterminate Potential): potent and newly recognized contributor to cardiovascular risk. Circulation 138 666-668 (2018)

28. Calvillo-Argüelles, O. et al. Connections between clonal hematopoiesis, cardiovascular disease, and cancer: a review. JAMA Cardiol. 4, 380 (2019).

29. Bolton, K. L. et al. Managing clonal hematopoiesis in patients with solid tumors. J. Clin. Oncol. 37, 7-11 (2019).

30. Campbell, I. M., Shaw, C. A., Stankiewicz, P. \& Lupski, J. R. Somatic mosaicism: implications for disease and transmission genetics. Trends Genet. TIG 31, 382-392 (2015).

31. Forsberg, L. A., Gisselsson, D. \& Dumanski, J. P. Mosaicism in health and disease - clones picking up speed. Nat. Rev. Genet. 18, 128-142 (2017).

32. Konnick, E. Q. \& Pritchard, C. C. Germline, hematopoietic, mosaic, and somatic variation: interplay between inherited and acquired genetic alterations in disease assessment. Genome Med. 8, (2016).
33. Steensma, D. P. Clinical consequences of clonal hematopoiesis of indeterminate potential. Blood Adv. 2, 3404-3410 (2018).

34. Acuna-Hidalgo, R. et al. Ultra-sensitive sequencing identifies high prevalence of clonal hematopoiesis-associated mutations throughout adult life. Am. J. Hum. Genet. 101, 50-64 (2017).

35. McNulty, S. N. et al. Diagnostic utility of next-generation sequencing for disorders of somatic mosaicism: a five-year cumulative cohort. Am. J. Hum. Genet. 105, 734-746 (2019).

36. Tumor evolution. High burden and pervasive positive selection of somatic mutations in normal human skin. - Abstract - Europe PMC. https://europepmc. org/article/MED/25999502.

37. Yizhak, K. et al. RNA sequence analysis reveals macroscopic somatic clonal expansion across normal tissues. Science 364, (2019).

38. Hernando, B. et al. The effect of age on the acquisition and selection of cancer driver mutations in sun-exposed normal skin. Ann. Oncol. 32, 412-421 (2021).

39. Bougeard, G. et al. Revisiting Li-fraumeni syndrome from TP53 mutation carriers. J. Clin. Oncol. 33, 2345-2352 (2015).

40. Genovese, G. et al. Clonal hematopoiesis and blood-cancer risk inferred from blood DNA sequence. N. Engl. J. Med. 371, 2477-2487 (2014).

41. Guermouche, $\mathrm{H}$. et al. High prevalence of clonal hematopoiesis in the blood and bone marrow of healthy volunteers. Blood Adv. 4, 3550-3557 (2020).

42. Midic, D. et al. Prevalence and dynamics of clonal hematopoiesis caused by leukemia-associated mutations in elderly individuals without hematologic disorders. Leukemia 34, 2198-2205 (2020).

\section{ACKNOWLEDGEMENTS}

The authors declare no funding for this research from any funding agency in the public, commercial or not-for-profit sectors. R.H.K. is supported by the Bhalwan Family Chartiable Foundation.

\section{AUTHOR CONTRIBUTIONS}

R.H.K. and H.L. conceptualized the project. R.H.K., N.M., H.L., and K.M.F., planned the manuscript. R.H.K. and N.M. drafted and wrote the manuscript. K.M.F. and H.L. assisted with writing of the manuscript. H.L., P.A., K.P., K.B. and K.S. provided the raw clinical data. K.M.F. and J.M.M. assisted with data curation. R.H.K. and N.M. analyzed and compiled clinical data. N.M., K.P., J.M.M., K.B., K.S., K.M.F., P.A., S.P., S.R.A., H.L., B.S., and R.H.K. reviewed and provided substantial edits to each manuscript draft. R.H.K., N. M., and K.M.F. submitted the manuscript for publication.

\section{COMPETING INTERESTS}

The authors declare no competing interests.

\section{ADDITIONAL INFORMATION}

Supplementary information The online version contains supplementary material available at https://doi.org/10.1038/s41525-021-00224-6.

Correspondence and requests for materials should be addressed to H.L. or R.H.K

Reprints and permission information is available at http://www.nature.com/ reprints

Publisher's note Springer Nature remains neutral with regard to jurisdictional claims in published maps and institutional affiliations.

\begin{abstract}
Open Access This article is licensed under a Creative Commons Attribution 4.0 International License, which permits use, sharing, adaptation, distribution and reproduction in any medium or format, as long as you give appropriate credit to the original author(s) and the source, provide a link to the Creative Commons license, and indicate if changes were made. The images or other third party material in this article are included in the article's Creative Commons license, unless indicated otherwise in a credit line to the material. If material is not included in the article's Creative Commons license and your intended use is not permitted by statutory regulation or exceeds the permitted use, you will need to obtain permission directly from the copyright holder. To view a copy of this license, visit http://creativecommons.org/licenses/by/4.0/.
\end{abstract}

(c) The Author(s) 2021 